Revista Tecnologia e Ambiente, v. 21, n.1, 2015, Criciúma, Santa Catarina. ISSN: 1413-8131 (versão impressa) ISSN: 2358-9426 (versão eletrônica)

\title{
REGISTRO DE IMAGEM DIGITAL EFETUADO POR MEIO DE MODELOS POLINOMIAIS
}

\section{REGISTRATION OF DIGITAL IMAGE PERFORMED WITH POLYNOMIAL MODEL}

\author{
Nilzo Ivo Ladwig ${ }^{1}$ \\ Leonard Niero da Silveira ${ }^{2}$ \\ Diego Medeiros ${ }^{3}$
}

\begin{abstract}
RESUMO
O registro de uma imagem digital (genericamente denominada de georreferenciamento pelos profissionais da área da geomensura) é a transformação geométrica na qual se relaciona as coordenadas da imagem com as coordenadas geodésicas ou geográficas, sendo estas esferoidais ou planas (projeção cartográfica). O artigo tem como objetivo o registro de imagem digital utilizando modelos polinomiais de $1^{\mathrm{a}}, 2^{\mathrm{a}}$ e $3^{\mathrm{a}}$ ordens. Foram definidos 36 pontos rastreados com receptores GNSS marca Geomax, modelo Zenith 25 e processados com os softwares Geomax geo Office e Topcon Tools. O software utilizado para o registro da imagem foi o Quantum GIS (QGIS) versão 2.4. Os resultados demonstraram que os modelos polinomiais apresentam suas restrições para o modelamento dos pontos, o de $1^{\mathrm{a}}$ ordem foi que apresentou os melhores resultados entre as três ordens utilizadas na quantidade de pontos com pixels residuais inferior a 1 (um) não conseguindo ajustar todos. Conclui-se que o produto cartográfico em formato raster registrado pode ser trabalhado em uma escala maior que 1:10000 em se tratando de planimetria conforme consta no DECRETO $\mathrm{N}^{\circ}$ 89.817 DE 20 DE JUNHO DE 1984 que estabelece as Instruções Reguladoras das Normas Técnicas da Cartografia Nacional.
\end{abstract}

PALAVRAS-CHAVE: Georreferenciamento; Geodésia; Cartografia; Fotogrametria.

\section{ABSTRACT}

The registration of a digital image (generally called georeferencing by the land surveying professionals) is the geometric transformation in which relates the image with geodetic coordinates or geographical coordinates, which are spheroidal or flat (map projection). The article aims at the digital image registration using polynomial models of 1st, 2nd and 3rd orders, 36 points have been defined traced with GNSS receivers Geomax - Zenith25 model and processed with Geomax geo Office and Topcon Tools software. The software used to record image was the Quantum GIS (QGIS) Version 2.4. The results showed that the polynomial models have their limitations for modeling of the points, the 1st order was that showed the best results among the three courses used in many points with residual pixels less

\footnotetext{
${ }^{1}$ Professor do Curso de Engenharia de Agrimensura e Programa de Pós Graduação em Ciências Ambientais UNESC (Universidade do Extremo Sul Catarinense), Professor da UNISUL (Universidade do Sul de Santa Catarina), e mail: ladwig@unesc.net

${ }^{2}$ Professor do Curso de Engenharia de Agrimensura - UNESC (Universidade do Extremo Sul Catarinense), email: leonard@amiranet.com.br

3 Engenheiro Agrimensor, UNESC (Universidade do Extremo Sul Catarinense), e-mail:
} diegomedeiros92@hotmail.com 
Revista Tecnologia e Ambiente, v. 21, n.1, 2015, Criciúma, Santa Catarina. ISSN: 1413-8131 (versão impressa) ISSN: 2358-9426 (versão eletrônica)

than one (1) failing to adjust all. We conclude that the cartographic product registered raster can be worked on a larger scale than 1:10000 in the case of planimetry.

KEYWORDS: Georeferencing; Geodesy; Cartography; Photogrammetry.

\section{Introdução}

O trabalho tem como objetivo o georreferenciamento de imagem digital utilizando modelos polinomiais que tem como benefício a fácil utilização e não necessita o conhecimento dos parâmetros do sistema imageador, resultando em um produto cartográfico em formato raster corrigido geometricamente.

Para a realização desse trabalho se fez necessário utilizar os conceitos de três ciências, a geodésia, a cartografia e a fotogrametria.

A contribuição da geodésia para o georreferenciamento de imagem é fornecer o posicionamento global pelo sistema GNSS (Sistema de Navegação Global por Satélite), obtendo-se as coordenadas dos GCP's (Ground Control Points), com trabalho de campo e processamento em ambiente computacional.

De acordo com ZANETTI (2007) Geodésia é o estudo dos métodos e técnicas utilizadas para a determinação das dimensões e formas da terra, considerando o campo de gravidade que influencia nas determinações e medições. O mesmo também é determinado por essa ciência, por meio de observações determinando coordenadas de pontos na superfície terrestre.

Segundo o INCRA (Instituto Nacional de Colonização e Reforma Agrária, 2013) o posicionamento GNSS pode ser realizado com diversos métodos e procedimentos, com foco em georreferenciamento de imóveis rurais, mas também serve para realização de trabalhos que necessitam da utilização do sistema de satélites global e determinação de coordenadas, vértices de referencia.

A área da cartografia é aplicada no conhecimento dos sistemas de projeção cartográfica.

Diferentes projeções cartográficas foram desenvolvidas para permitir a representação da esfericidade terrestre num plano (mapas e cartas), cada uma priorizando determinado aspecto da representação (dimensão, forma, etc.). (IBGE, 2012, p.21) 
Revista Tecnologia e Ambiente, v. 21, n.1, 2015, Criciúma, Santa Catarina. ISSN: 1413-8131 (versão impressa) ISSN: 2358-9426 (versão eletrônica)

Sabe-se que a terra é irregular e é impossível representá-la em uma superfície plana sem apresentar algumas distorções, por isso, vários métodos foram desenvolvidos para representar uma área de interesse em um mapa e minimizar ao máximo os efeitos da curvatura terrestre, denominando-se de sistemas de projeção cartográfica, uma delas o sistema UTM (Universal Transversa de Mercator).

O sensoriamento remoto, principalmente no que concerne aos sensores suborbitais (como a aerofotogrametria, por exemplo), tem como objetivo a obtenção de dados precisos de imagens podendo ser verticais ou horizontais.

Segundo ANDRADE (2003) a fotogrametria tem sua maior aplicação na produção de mapas com apoio de outras ciências como a geodésia e cartografia.

Para a utilização da técnica digital utilizam-se computadores com grande capacidade de processamento com hardware e software, específico exigido devido a quantidade de informações a ser processada. A principal aplicação da fotogrametria no trabalho foi realizar a correção geométrica utilizando métodos de transformação polinomial.

\section{Materiais e métodos}

Para a realização do georreferenciamento de imagem digital com modelos polinomiais, foi definida a área de interesse localizada dentro do perímetro urbano do município de Cocal do Sul, Estado de Santa Catarina. Visando atender o objetivo de georreferenciamento elegeu-se uma área densamente edificada para garantir uma homogeneização dos pontos de controle/checagem e uma maior quantidade de feições definidas resultantes da urbanização, facilitando a identificação na imagem e posterior rastreio pelo sistema GNSS dos mesmos.

Segundo CROSTA (1992) é necessário identificar de 6 a 10 pontos de controle no mínimo em uma imagem de 1000 x 1000 pixels para realizar correções na imagem e a margem de erro recomendável deve ser de 1 pixel. Consequentemente quanto maior for a quantidade de pixels, a de GCP's proporcionalmente, também será.

Definiu-se uma quantidade de 36 pontos para que todos estivessem bem distribuídos e que formassem uma rede homogênea em toda imagem, objetivando um melhor resultado do georreferenciamento. Todas as feições escolhidas para o rastreio foram baseadas na definição visual na imagem, e todos os alvos físicos são entroncamentos de muros. 
Revista Tecnologia e Ambiente, v. 21, n.1, 2015, Criciúma, Santa Catarina. ISSN: 1413-8131 (versão impressa) ISSN: 2358-9426 (versão eletrônica)

No dia do levantamento dos pontos em campo, 5 deles foram excluídos, o ponto 3 devido a propriedade estar fechada e sem a presença do proprietário na mesma, os pontos 11 e 14 devido o entroncamento dos muros serem arredondados, o 15 que devido a interferência do sinal da antena de celular que estava próxima apresentava na coletora a solução flutuante, o 20 foi descartado devido a proximidade com o ponto 19.

O equipamento utilizado foi um par de receptores GNSS marca Geomax, modelo Zenith25 e os softwares de processamento utilizados foram o Geomax geo Office e Topcon Tools, onde o primeiro software citado serviu para obtenção dos dados de rastreio e transformação dos mesmos em extensão RINEX (Receiver Independent Exchange), para posteriormente ser importado no Topcon Tools com a efeméride precisa obtida via internet no site IGS - International GNSS Service para o dia 21/10/2014 quando foram realizadas as observações, juntamente com a RBMC de Imbituba para triangulação com as bases e pontos rastreados.

O método de levantamento utilizado foi o relativo estático rápido, com tempo de ocupação de 5 minutos do equipamento móvel (Rover), com linha de base inferior a 2500 metros.

O software utilizado para o registro da imagem foi o Quantum GIS (QGIS) versão 2.4, sendo um programa de fácil utilização, intuitivo, de código aberto, além de sofrer constantes atualizações com a interface com o usuário em português do Brasil. A principal característica que foi levada em consideração para a escolha do software, foi que o mesmo possui a visualização das discrepâncias entre as coordenadas iniciais e as finais dos pontos de controle, e o erro médio quadrático são apresentados em pixels residuais. A ferramenta do software para a realização do trabalho chama-se Georreferenciador localizada na aba raster do layout inicial. Neste espaço insere-se o raster que se deseja trabalhar e nele localizam-se as feições/GPC com auxilio do periférico (mouse) que foram definidas anteriormente pelo estudo da imagem, cada uma delas possui coordenada que são inseridas individualmente, e posteriormente se atribui o X e Y rastreados pelo sistema GNSS, e para a etapa final define-se o método de transformação e o método de reamostragem.

\section{Analise dos Resultados}


Revista Tecnologia e Ambiente, v. 21, n.1, 2015, Criciúma, Santa Catarina. ISSN: 1413-8131 (versão impressa) ISSN: 2358-9426 (versão eletrônica)

Área de estudo (imagem raster) está localizada no perímetro urbano do município de Cocal do Sul, Estado de Santa Catarina. A figura 1 que segue mostra a área de estudo com os GPC's (Ground Control Points) utilizados no processamento dos dados.

Figura 1 - Área de estudo com a distribuição dos GPC's (Ground Control

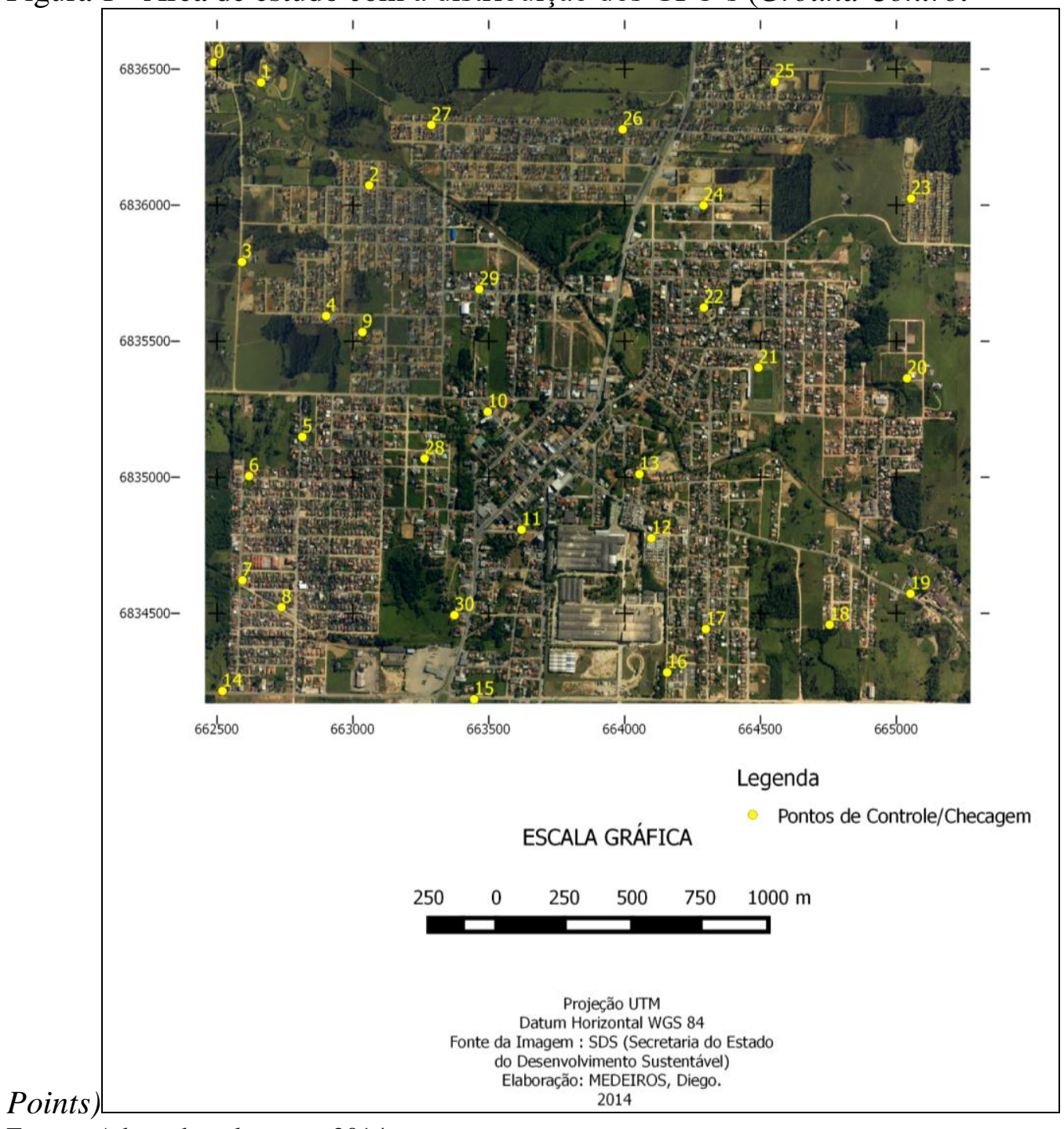

Fonte - Adaptado pelo autor, 2014.

Na tabela 1 podem ser observadas as coordenadas finais de solução fixa, trabalhadas no datum WGS 84, após o processamento das mesmas no software Topcon Tools, e na Figura 2 a solução fixa das observações com as precisões horizontal e vertical, o tempo de ocupação e o método de Ponto Preciso.

Tabela 1 - Coordenadas finais dos GPC's \begin{tabular}{lll}
\hline Ponto & Coordenadas X (m) & Coordenadas Y (m) \\
\hline
\end{tabular} 
Revista Tecnologia e Ambiente, v. 21, n.1, 2015, Criciúma, Santa Catarina. ISSN: 1413-8131 (versão impressa) ISSN: 2358-9426 (versão eletrônica)

\begin{tabular}{lll}
\hline $\mathbf{0}$ & 662489,786 & 6836524,976 \\
$\mathbf{1}$ & 662661,987 & 6836451,23 \\
$\mathbf{2}$ & 663060,042 & 6836072,929 \\
$\mathbf{3}$ & 662592,098 & 6835791,598 \\
$\mathbf{4}$ & 662901,584 & 6835592,841 \\
$\mathbf{5}$ & 662814,015 & 6835148,349 \\
$\mathbf{6}$ & 662617,909 & 6835004,18 \\
$\mathbf{7}$ & 662593,506 & 6834621,344 \\
$\mathbf{8}$ & 662737,274 & 6834522,102 \\
$\mathbf{9}$ & 663035,226 & 6835533,475 \\
$\mathbf{1 0}$ & 663496,241 & 6835240,668 \\
$\mathbf{1 1}$ & 663620,854 & 6834807,336 \\
$\mathbf{1 2}$ & 664098,794 & 6834775,771 \\
$\mathbf{1 3}$ & 664054,614 & 6835011,468 \\
$\mathbf{1 4}$ & 662520,194 & 6834214,273 \\
$\mathbf{1 5}$ & 663446,417 & 6834183,851 \\
$\mathbf{1 6}$ & 664156,75 & 6834282,632 \\
$\mathbf{1 7}$ & 664299,076 & 6834442,054 \\
$\mathbf{1 8}$ & 664754,851 & 6834457,694 \\
$\mathbf{1 9}$ & 665052,165 & 6834571,82 \\
$\mathbf{2 0}$ & 665039,849 & 6835363,578 \\
$\mathbf{2 1}$ & 664492,594 & 6835402,989 \\
$\mathbf{2 2}$ & 664291,923 & 6835623,645 \\
$\mathbf{2 3}$ & 665054,388 & 6836024,255 \\
$\mathbf{2 4}$ & 664290,49 & 6835999,332 \\
$\mathbf{2 5}$ & 664552,511 & 6836453,405 \\
$\mathbf{2 6}$ & 663993,001 & 6836278,614 \\
$\mathbf{2 7}$ & 663288,958 & 6836294,667 \\
$\mathbf{2 8}$ & 663264,17 & 6835068,5 \\
$\mathbf{2 9}$ & 663464,959 & 6835691,091 \\
$\mathbf{3 0}$ & 663373,939 & 6834493,21 \\
\hline Fonte & $\mathrm{6}$ & \\
\hline
\end{tabular}

Fonte - Autor, 2014.

Figura 2: Solução fixa das observações no software Topcon Tools

\begin{tabular}{|c|c|c|c|c|c|c|}
\hline i... & $\Delta \quad$ Do Ponto & Duração & Precisão Horiz... & Precisão Vertic... & Método & Tipo Solução \\
\hline 9 & 1 & 0:05:01 & 0,001 & 0,002 & PP & Fixo \\
\hline 9. & 2 & 0:05:01 & 0,001 & 0,002 & PP & Fixo \\
\hline 9. & 4 & 0:05:02 & 0,003 & 0,005 & PP & Fixo \\
\hline 9 & 5 & 0:05:00 & 0,002 & 0,004 & Pp & Fixo \\
\hline 9 & 6 & 0:05:01 & 0,003 & 0,004 & PP & Fixo \\
\hline 9. & 7 & 0:05:01 & 0,002 & 0,003 & Pp & Fixo \\
\hline 9. & 8 & 0:05:01 & 0,003 & 0,005 & PP & Fixo \\
\hline s, & 9 & 0:05:01 & 0,004 & 0,007 & PP & Fixo \\
\hline Q & 10 & 0:05:01 & 0,006 & 0,014 & PP & Fixo \\
\hline 9 & 12 & 0:05:02 & 0,003 & 0,005 & PP & Fixo \\
\hline 9 & 13 & 0:05:00 & 0,003 & 0,005 & PP & Fixo \\
\hline 9. & 16 & 0:05:01 & 0,017 & 0,025 & Pp & Fixo \\
\hline 9, & 17 & 0:05:01 & 0,012 & 0,016 & PP & Fixo \\
\hline s. & 18 & 0:05:01 & 0,004 & 0,006 & PP & Fixo \\
\hline 9 & 19 & 0:05:01 & 0,002 & 0,005 & PP & Fixo \\
\hline 9 & 21 & 0:05:00 & 0,004 & 0,005 & PP & Fixo \\
\hline 9. & 22 & 0:05:01 & 0,023 & 0,036 & PP & Fixo \\
\hline 9. & 23 & 0:05:00 & 0,004 & 0,008 & Pp & Fixo \\
\hline 9. & 24 & 0:05:01 & 0,003 & 0,006 & PP & Fixo \\
\hline 9. & 25 & 0:05:00 & 0,003 & 0,005 & PP & Fixo \\
\hline 9. & 26 & 0:05:00 & 0,012 & 0,019 & PP & Fixo \\
\hline 9 & 27 & 0:05:00 & 0,002 & 0,004 & PP & Fixo \\
\hline 9, & 28 & 0:05:00 & 0,005 & 0,009 & PP & Fixo \\
\hline 9 & 29 & 0:05:01 & 0,007 & 0,013 & PP & Fixo \\
\hline 9. & 30 & 0:05:00 & 0,023 & 0,082 & PP & Fixo \\
\hline 9 & 31 & 0:05:01 & 0,015 & 0,029 & PP & Fixo \\
\hline 9. & 32 & 0:05:02 & 0,002 & 0,005 & PP & Fixo \\
\hline 9 & 33 & 0:04:57 & 0,004 & 0,006 & PP & Fixo \\
\hline 9 & 34 & 0:05:01 & 0,003 & 0,005 & PP & Fixo \\
\hline 9. & 35 & 0:05:00 & 0,003 & 0,005 & PP & Fixo \\
\hline 8 & 36 & 0:05:01 & 0,004 & 0,006 & PP & Fixo \\
\hline
\end{tabular}


Revista Tecnologia e Ambiente, v. 21, n.1, 2015, Criciúma, Santa Catarina. ISSN: 1413-8131 (versão impressa) ISSN: 2358-9426 (versão eletrônica)

Fonte - Autor, 2014.

\section{Descrição e análise dos resultados decorrentes da utilização dos modelos polinomiais}

A utilização dos modelos polinomiais levou em consideração o aspecto da imagem, quanto mais distorcida ela for, maior será o grau do polinômio necessário para a correção, e consequentemente a quantidade de GPC's aumenta. A quantificação do grau de distorção da imagem pode ser determinada a partir do primeiro processo de registro, por meio dos resíduos obtidos ou comparando as coordenadas reais dos GPC's com as coordenadas obtidas da imagem.

Equações polinomiais são utilizadas para converter as coordenadas do arquivo fonte para corrigir as coordenadas do mapa. Dependendo do grau de distorção da imagem, o número dos GCP's utilizados aumentam consequentemente as equações polinomiais se tornam mais complexas (grau do polinômio maior). O grau de complexidade do polinômio é expresso como a ordem do polinômio. A ordem é simplesmente o mais alto expoente usado no polinômio. (ERDAS, 1999. p.349).

Para a realização do registro o software calcula a matriz de transformação a partir dos pontos de controle/checagem. Os coeficientes obtidos são utilizados nas equações polinomiais para a conversão das coordenadas. $\mathrm{O}$ objetivo do calculo desses coeficientes é a derivação do polinômio para que se alcance o menor erro possível para transformar as coordenadas da imagem para um sistema de projeção conhecido.

Conforme determina ERDAS (1999) a quantidade mínima de pontos de controle para os métodos polinomiais seguem a equação abaixo.

Equação 1 - Quantidade de GCP's

GCPs $=\frac{((t+1) *(t+2))}{2}$

Onde:

$t$ é a ordem do polinômio

A relação matemática para a conversão depende dos coeficientes dos polinômios que são definidos pelos pontos de controle na imagem, e para cada ponto inserido ou retirado os coeficientes são modificados, conforme a necessidade do ajuste. 
Revista Tecnologia e Ambiente, v. 21, n.1, 2015, Criciúma, Santa Catarina. ISSN: 1413-8131 (versão impressa) ISSN: 2358-9426 (versão eletrônica)

Segundo ERDAS (1999) a matriz de transformação do polinômio de $1^{\text {a }}$ ordem possui 6 coeficientes, 3 para $\mathrm{X}$ e 3 para $\mathrm{Y}$, abaixo segue a matriz.

Matriz 1 - Matriz de transformação do polinômio de $1^{\text {a }}$ ordem

$\begin{array}{lll}a_{0} & a_{1} & a_{2}\end{array}$

$\begin{array}{lll}b_{0} & b_{1} & b_{2}\end{array}$

Os coeficientes são usados na equação do polinômio de $1^{\mathrm{a}}$ ordem, conforme equação que segue.

Equação 2 - Polinômio de $1^{\mathrm{a}}$ ordem

$X_{0}=a_{0}+a_{1} x+a_{2} y$

$Y_{0}=b_{0}+b_{1} x+b_{2} y$

Onde:

$\mathrm{X}$ e $\mathrm{Y}$ são as coordenadas iniciais

$\mathrm{X}_{0}$ e $\mathrm{Y}_{0}$ são as coordenadas retificadas

Equação 3 - Polinômio de $2^{\mathrm{a}}$ ordem

$X o=a_{0}+a_{1} x+a_{2} y+a_{3} x y+a_{4} x^{2}+a_{5} y^{2}$

$Y_{o}=b_{0}+b_{1} x+b_{2} y+b_{3} x y+b_{4} x^{2}+b_{5} y^{2}$

A quantidade de coeficientes da equação aumenta conforme a ordem do polinômio, quanto maior a ordem mais coeficientes serão necessários. Segue abaixo uma formula simples de se calcular a quantidade de coeficientes conforme a ordem da equação.

Equação 4 - Quantidade de coeficientes conforme a ordem polinomial

$(t+1) *(t+2)$

Onde:

$t$ é o grau do polinômio

O método de reamostragem do Vizinho mais Próximo utiliza o valor mais próximo do centro do pixel antigo, para o valor central do novo pixel, a grande vantagem deste método é que ele mantém os valores inalterados, que é importante para o processamento e classificação.

Conforme TEEUW et al (2005), para o cálculo do erro é utilizado o método do Erro Médio Quadrático, onde verifica-se a diferença entre o valor da observação e o valor estimado para cada ponto definido. Acha-se o valor médio quadrado e após encontra-se a raiz do 
Revista Tecnologia e Ambiente, v. 21, n.1, 2015, Criciúma, Santa Catarina. ISSN: 1413-8131 (versão impressa) ISSN: 2358-9426 (versão eletrônica)

mesmo. Os valores RMS (Erro Médio Quadrático) são definidos conforme o tamanho do pixel da imagem. Segue abaixo a equação.

Equação 5 - Erro médio quadrático

$R M S=\sqrt{\sum(X s-X c)^{2}+(Y s-Y c)^{2}}$

Onde:

Xs e Ys são as coordenadas das observações e Xc e Yc são as coordenadas iniciais

A tabela 2 que segue mostra os resultados da relação dos modelos polinomiais com os 30 pontos e os valores residuais dos pixels.

Tabela 2: Relação do modelo polinomial com os pontos e os valores residuais dos pixels

\begin{tabular}{|c|c|c|c|}
\hline ID & $\begin{array}{l}\text { Residual } \\
\left(\text { Pixels) } 1^{\mathrm{a}}\right. \\
\text { Ordem }\end{array}$ & $\begin{array}{l}\text { Residual } \\
\text { (Pixels) } 2^{\mathrm{a}} \\
\text { Ordem }\end{array}$ & $\begin{array}{l}\text { Residual } \\
\text { (Pixels) } 3^{\mathrm{a}} \\
\text { Ordem }\end{array}$ \\
\hline 0 & 2.31 & 1.91 & 2.17 \\
\hline 1 & 0.79 & 0.95 & 1.27 \\
\hline 2 & 0.56 & 0.94 & 0.95 \\
\hline 3 & 3.55 & 3.52 & 3.51 \\
\hline 4 & 1.34 & 1.19 & 0.96 \\
\hline 5 & 1.24 & 1.18 & 1.58 \\
\hline 6 & 0.97 & 1.33 & 0.96 \\
\hline 7 & 0.24 & 1.07 & 1.50 \\
\hline 8 & 0.53 & 1.02 & 1.06 \\
\hline 9 & 2.11 & 2.31 & 1.05 \\
\hline 10 & 0.70 & 0.98 & 1.29 \\
\hline 11 & 1.16 & 1.21 & 1.35 \\
\hline 12 & 1.85 & 1.70 & 1.62 \\
\hline 13 & 1.42 & 1.14 & 1.28 \\
\hline 14 & 0.98 & 0.36 & 0.70 \\
\hline 15 & 0.75 & 0.91 & 1.33 \\
\hline 16 & 2.50 & 2.66 & 3.06 \\
\hline 17 & 0.31 & 0.56 & 0.67 \\
\hline 18 & 2.20 & 1.87 & 2.08 \\
\hline 19 & 3.08 & 3.13 & 3.08 \\
\hline 20 & 0.97 & 0.56 & 0.96 \\
\hline 21 & 1.31 & 1.32 & 1.53 \\
\hline 22 & 0.52 & 0.47 & 0.50 \\
\hline 23 & 3.29 & 2.04 & 2.01 \\
\hline 24 & 4.20 & 4.35 & 4.35 \\
\hline 25 & 2.31 & 1.79 & 2.11 \\
\hline
\end{tabular}


Revista Tecnologia e Ambiente, v. 21, n.1, 2015, Criciúma, Santa Catarina. ISSN: 1413-8131 (versão impressa) ISSN: 2358-9426 (versão eletrônica)

\begin{tabular}{llll}
26 & 1.19 & 1.13 & 1.12 \\
27 & 1.55 & 1.11 & 1.16 \\
28 & 2.65 & 2.52 & 2.68 \\
29 & 2.21 & 2.46 & 2.71 \\
30 & 0.85 & 1.01 & 1.03 \\
\hline
\end{tabular}

A transformação polinomial de $1^{\text {a }}$ ordem é o método de correção geométrica linear que permite o escalonamento, rotação e translação/distorção do arquivo raster a ser trabalhado, preservando a colinearidade.

Nos resultados deste método pode-se observar que existem 12 pontos com pixels residuais inferior a 1 (um), e os 19 pontos restantes apresentaram resíduo superior a 1 (um) pixel, onde o maior erro médio quadrático foi no ponto 24 com 4,20 pixels, destacado acima na tabela 2.

$\mathrm{Na}$ transformação polinomial de $2^{\mathrm{a}}$ ordem que é um método de correção geométrica não linear, que é utilizado para converter uma imagem influenciada pela curvatura terrestre em uma projeção plana, ou grandes áreas imageadas que também sofrem influencia da curvatura e relevo.

Os resultados deste método mostraram que existem 8 pontos com pixels residuais inferior a 1 (um), o mais preciso foi o ponto de numero 14 com 0,36 pixels de erro médio quadrático, e o restante dos 23 pontos apresentaram erros superiores a 1(um) pixel, destaque para o ponto 24 que apresentou 4,35 de pixel residual.

Por último a transformação polinomial de $3^{\mathrm{a}}$ ordem é um método de correção geométrica não linear, é utilizada para imagens com graves distorções, tomadas Off nadir, como imagens suborbitais de alta resolução.

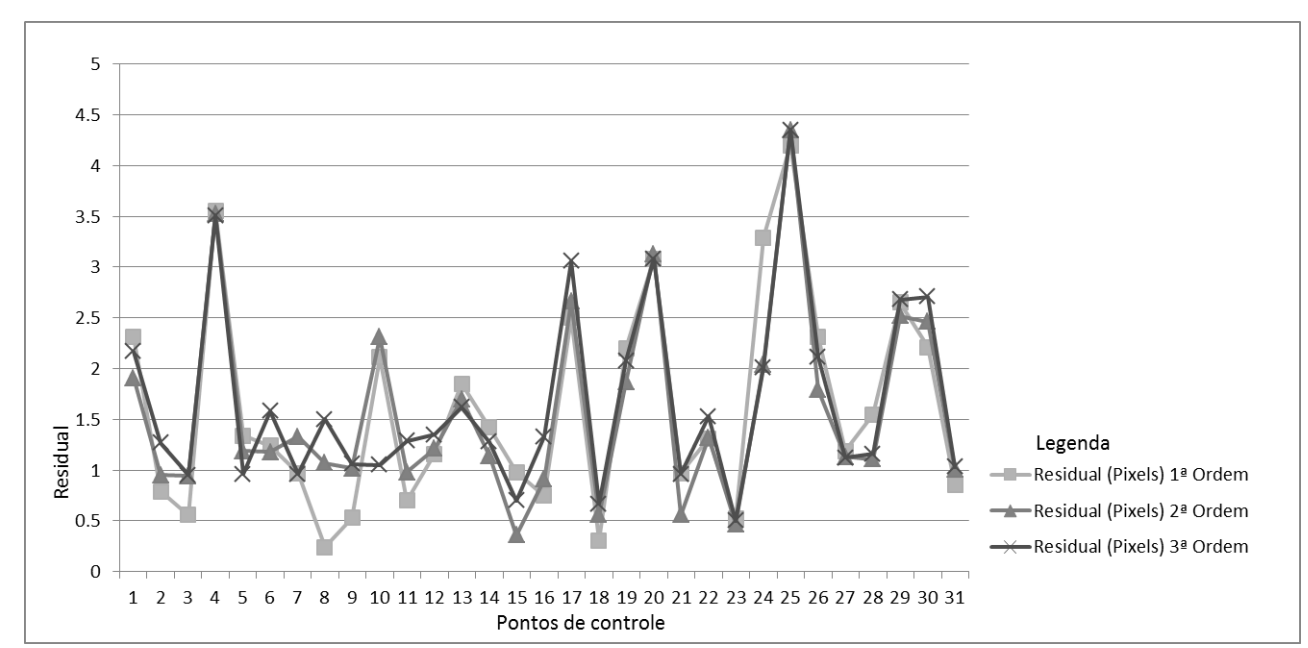


Revista Tecnologia e Ambiente, v. 21, n.1, 2015, Criciúma, Santa Catarina. ISSN: 1413-8131 (versão impressa) ISSN: 2358-9426 (versão eletrônica)

Figura 3: Distribuição dos residuais nos 3 métodos de correção geométrica Fonte: Autor, 2014

Neste método pode-se observar que existem 6 pontos com pixels residuais inferior a 1 (um), o ponto mais preciso desse método foi o 22 com 0,5 pixel de erro médio quadrático, e o restante dos 25 pontos acima de 1 (um) pixel, destacando-se o de número 24 com 4,35 residuais.

Os resultados das transformações geométricas seguem as características da ordem dos polinômios adotados, a transformação de $1^{\text {a }}$ ordem é linear utilizada satisfatoriamente em locais imageados que tem relevo plano e pouco ondulado, as transformações de $2^{\mathrm{a}}$ e $3^{\mathrm{a}}$ ordem são não lineares utilizadas para imagens com distorções de relevo e curvatura terrestre.

Conforme a complexidade do polinômio aumenta, a curva de ajuste que deve ser mais próxima dos pontos. A transformação AFIM pode subestimar as distorções existentes, porém as transformações não lineares podem provocar distorções no produto gerado, ajustando alguns pontos e modelando erros de outros, por esse motivo os polinômios mais complexos só devem ser aplicados quando o raster necessita de uma maior modelação geométrica para a realização da correção.

A imagem utilizada é proveniente do produto aerofotogramétrico disponibilizado pela SDS (Secretaria de Estado do Desenvolvimento Sustentável de Santa Catarina), então o raster trabalhado, já havia passado por correção geométrica, apresentando escala homogênea e coordenadas georreferenciadas no sistema WGS 84.

Abaixo seguem dois quadros explicativos, o primeiro realiza o comparativo entre os métodos de transformação destacando os pontos fortes e fracos, o segundo destaca os pontos fortes e fracos do método de reamostragem utilizando o de Vizinho mais Próximo.

Quadro 1 - Comparativo entre os métodos de transformação.

\begin{tabular}{|c|c|c|}
\hline $\begin{array}{c}\text { Modelos para } \\
\text { Transformação }\end{array}$ & Pontos Fortes & Pontos Fracos \\
\hline Polinomial de $1^{\mathrm{a}}$ ordem & $\begin{array}{lc}\text { Preserva colinearidade, é } \\
\text { linear necessitando de } \\
\text { menos pontos } & \text { de } \\
\text { controle/checagem. } & \end{array}$ & $\begin{array}{l}\text { Não permite curvaturas e } \\
\text { diferenças de relevo. }\end{array}$ \\
\hline Polinomial de $2^{\mathrm{a}}$ ordem & $\begin{array}{l}\text { Curvaturas } \text { Moderadas e } \\
\text { distorções por diferenças } \\
\text { de relevo. }\end{array}$ & $\begin{array}{l}\text { Necessita de maior quantidade } \\
\text { de GPC's e pode introduzir } \\
\text { distorções em algumas situações. }\end{array}$ \\
\hline Polinomial de $3^{\mathrm{a}}$ ordem & Grandes $\quad$ Curvaturas $\mathrm{e}$ & Transformação complexa de \\
\hline
\end{tabular}




\begin{tabular}{|l|l|l|}
\hline & distorções. & $\begin{array}{l}\text { ajustamento para a obtenção de } \\
\text { bons resultados. }\end{array}$ \\
\hline
\end{tabular}

Fonte: Autor, 2014

Quadro 2 - Características do método de reamostragem.

\begin{tabular}{|l|l|l|}
\hline Método de Reamostragem & \multicolumn{1}{|c|}{ Pontos Fortes } & \multicolumn{1}{|c|}{ Pontos Fracos } \\
\hline Vizinho mais Próximo & $\begin{array}{l}\text { Mantém os níveis de cinza, } \\
\text { rápido processamento e fácil } \\
\text { utilização. }\end{array}$ & $\begin{array}{l}\text { Pode causar linhas } \\
\text { descontínuas, alguns valores } \\
\text { podem ser descartados } \\
\text { enquanto outros podem ser } \\
\text { duplicados. }\end{array}$ \\
\hline
\end{tabular}

Fonte: Autor, 2014

\section{CONSIDERAÇÕES FINAIS}

Com a quantidade de 31 GCP's a função polinomial de $1^{\text {a }}$ ordem foi o método que apresentou os melhores resultados entre as três ordens utilizadas, na quantidade de pontos com pixels residuais inferior a 1(um), devido a grande quantidade de pontos acima da recomendada para correções utilizando este modelo, que varia entre 6 a 10 GPC's, solução fixa e precisa das coordenadas, homogeneidade dos pontos na área de estudo.

A imagem utilizada já havia passado por um processo de correção geométrica e georreferenciamento oferecendo uma escala homogênea favorecendo o método, que é uma transformação linear da imagem para um sistema de projeção, porém de 31 pontos apenas 12 apresentaram resíduos inferiores a 1(um) pixel, verifica-se que os resultados dependeram da acurácia e precisão do operador no momento da inserção do GPC e da sua respectiva coordenada calculada, e existem diferenças de relevo em vários locais da área de estudo que influenciam negativamente nos resultados.

Os métodos de $2^{\mathrm{a}}$ e $3^{\mathrm{a}}$ ordem ficaram longe do resultado desejado, a quantidade de pontos com erro médio quadrático inferior a $1(\mathrm{um})$ pixel foram 8 para o de $2^{\mathrm{a}}$ ordem e 6 para o de $3^{\text {a }}$ ordem, o motivo para a diferença da quantidade de pontos com precisão abaixo de 1 pixel em relação a primeira transformação, é que a característica principal dos métodos de segundo e terceiro grau é o modelamento não linear, o raster trabalhado já estava corrigido geometricamente, a utilização dos mesmos, acabou implementando erros e distorções.

Pode-se concluir que os métodos apresentam suas restrições para o modelamento dos pontos, o de $1^{\mathrm{a}}$ ordem não consegue ajustar todos, alguns acabaram ficando longe da reta de ajustamento, e as curvas dos polinômios de $2^{\mathrm{a}}$ e $3^{\mathrm{a}}$ ordem também não conseguem modelar e ajustar todos os pontos. 
Revista Tecnologia e Ambiente, v. 21, n.1, 2015, Criciúma, Santa Catarina. ISSN: 1413-8131 (versão impressa) ISSN: 2358-9426 (versão eletrônica)

O ponto 24 que apresentou os piores resultados de pixel residual nos três métodos é importante para a modelagem dos pontos restantes. Um teste de processamento com a retirada do ponto 24 foi realizado nos três processos e a quantidade de pontos com resíduos superiores a 1(um) pixel aumentaram, piorando o resultado do trabalho, ressalta-se a necessidade da sua permanência para o registro.

Conforme os resultados obtidos, o produto cartográfico em formato raster pode ser trabalhado em uma escala maior que 1:10000 em se tratando de planimetria conforme consta no DECRETO No 89.817 DE 20 DE JUNHO DE 1984 que estabelece as Instruções Reguladoras das Normas Técnicas da Cartografia Nacional.

\section{REFERENCIAS BIBLIOGRÁFICAS}

ANDRADE, J. B. Fotogrametria. Curitiba; SBEE, $2^{\text {a }}$ edição, 2003, 274p.

CRÓSTA, A. P. Processamento digital de imagens de sensoriamento remoto. Campinas, SP: UNICAMP, 1992, 170p.

ERDAS, Inc. ERDAS Field Guide, 5th ed. ERDAS, Inc., Atlanta, Georgia USA. 1999, 653p.

IBGE. Atlas geográfico escolar. 6. ed., Rio de Janeiro: IBGE, 2012, 218p.

INCRA. Manual Técnico de Posicionamento: georreferenciamento de imóveis rurais. República Federativa do Brasil - Ministério do Desenvolvimento Agrário, Instituto Nacional de Colonização e Reforma Agrária, Diretoria de Ordenamento da Estrutura Fundiária Coordenação Geral de Cartografia - Brasília, 2013. 34p.

TEEUW, R.; WHITESIDE, M.; McWILlIAM, N.; ZUKOWSKYJ, P. Field Techniques: GIS, GPS and Remote Sensing. London. Published by Geography Outdoors - Royal Geographical Society (with IBG), 2005, 366p.

ZANETTI, M. A. Z. Geodésia. Universidade Federal do Paraná, Curitiba, Paraná, 2007, 92p. 